\title{
Article \\ Planning of Optical Connections in 5G Packet-Optical xHaul Access Network
}

\author{
Mirosław Klinkowski *(D) and Marek Jaworski (D) \\ National Institute of Telecommunications, Szachowa 1, 04-894 Warsaw, Poland; M.Jaworski@il-pib.pl \\ * Correspondence: M.Klinkowski@il-pib.pl
}

check for updates

Citation: Klinkowski, M.; Jaworski, M. Planning of Optical Connections in 5G Packet-Optical xHaul Access Network. Appl. Sci. 2022, 12, 1146. https://doi.org/10.3390/ app12031146

Academic Editors: Amalia Miliou and Fabio Cavaliere

Received: 30 November 2021

Accepted: 20 January 2022

Published: 22 January 2022

Publisher's Note: MDPI stays neutral with regard to jurisdictional claims in published maps and institutional affiliations.

Copyright: (c) 2022 by the authors. Licensee MDPI, Basel, Switzerland. This article is an open access article distributed under the terms and conditions of the Creative Commons Attribution (CC BY) license (https:/ / creativecommons.org/licenses/by/ $4.0 /)$.

\begin{abstract}
One of the main challenges in dense 5G radio access networks (RANs) is provisioning of low-cost connectivity between a large number of antennas, located at remote sites, and a central site (hub) in which baseband processing functions are performed. Packet-switched Ethernet and wavelength division multiplexing (WDM) are two principal transport network technologies enabling the reduction of the demand for direct optical fiber connections between the antennas and the hub. Whereas Ethernet allows for statistical multiplexing of multiple xHaul (fronthaul/midhaul/backhaul) flows and their aggregation in a high-capacity transmission link, WDM makes it possible to establish a number of such links (using different wavelengths) in a single optical fiber. Additional savings in the amount of fibers required can be achieved by means of optical add-drop multiplexers (OADMs) that allow for obtaining access to unused wavelengths by intermediate remote nodes, whenever the capacity on the WDM system is not fully utilized by the end remote node. In this work, we focus on the problem of planning optimal fiber connections, including the placement of OADMs for a set of wavelength demands at remote sites, with the aim of minimizing the amount of fibers used in a packet-optical xHaul access network carrying 5G traffic. We consider a passive WDM system in which the maximum transmission distance, estimated using an optical power-budget model, depends on the number of OADMs that are present on the transmission path. To formulate and solve the optimization problem, we make use of integer linear programming (ILP). We apply the ILP model in network analysis. In particular, by means of numerical experiments performed for two different network topologies, we study the impact of traffic load (in terms of the number of requested wavelengths) and optical multiplexer loss on the number of transmission paths that have to be established in the network. Obtained results show that the savings in fiber connections of up to $65 \%$ can be achieved in a packet-optical xHaul network if OADMs are used when compared to the scenario without OADMs.
\end{abstract}

Keywords: 5G networks; centralized radio access network; packet-switched xHaul network; optical network; WDM; network planning; network optimization; ILP modeling

\section{Introduction}

Telecommunication networking is undergoing a profound transformation related to the deployment of 5G networks [1]. This transformation is accompanied by evolution of the mobile network towards centralized and virtualized radio access network (C-RAN/vRAN) architectures [2]. Already in centralized 4G/long term evolution (LTE) network implementations, the base station has been disaggregated into a remote radio head (RRH), located close to the antenna at a remote site (cell), and a baseband unit (BBU) placed at a central site (hub). Distributed RRHs and centralized BBUs are connected using the common public radio interface (CPRI) protocol in such networks. In 5G, the radio frequency processing functions performed by a BBU are realized by a distributed unit (DU) and a central unit (CU), whereas RRH is replaced by a radio unit (RU), which performs low-level physical functions [3]. The DUs and CUs may be placed at different network locations in accordance with particular requirements of diverse $5 \mathrm{G}$ services, which can be classified 
as enhanced mobile broadband (eMBB), ultra-reliable and low-latency communications (URLLC), and massive machine-type communications (mMTC). For instance, the whole radio processing stack (i.e., DU/CU) might be placed at a remote site in mMTC applications, whereas the DU processing for eMBB services may be performed at a hub site, as mentioned in Reference [4]. The disaggregation and distributed placement of 5G RAN functions, results in multiple data flows that differ in terms of bandwidth and latency requirements. These flows, related to fronthaul ( $\mathrm{FH}$ - between RU and DU), midhaul ( $\mathrm{MH}$ - between DU and $\mathrm{CU}$ ), and backhaul (BH-between $\mathrm{CU}$ and a 5G core network) connections, should be carried using a convergent xHaul transport network, as mentioned in Reference [5]. In particular, the transport of traffic from remote sites and its aggregation into a hub site is realized through an xHaul access network.

5G networks are expected to make use of a much larger amount of installed antennas and access points than previous generations of mobile networks, which is frequently referred to as network densification. To decrease the capacity requirements of transport links in RANs, different techniques for compression of radio data have been proposed [6-8]. Still, the use of conventional transport solutions, based on dedicated point-to-point CPRI links, is neither scalable nor cost-effective in dense 5G access networks as it results in huge demand for high-bandwidth links between the antennas and the hub site. Therefore, to assure a convergent, scalable, and low-cost transport of radio traffic, the adaptation of well-known packet-based Ethernet technology has been proposed for xHaul transport networks in the IEEE standards 802.1CM [9] and 1914.1 [4]. Ethernet enables statistical multiplexing of data flows and, hence, increased utilization of link bandwidth. The encapsulation of $5 \mathrm{G}$ radio data into Ethernet frames is achieved by means of the enhanced CPRI (eCPRI) protocol [10]. The use of Ethernet in xHaul allows for support, as well other services not related to $5 \mathrm{G}$, such as legacy $4 \mathrm{G}$, enterprise, and residential services. In particular, the CPRI data related to $4 \mathrm{G}$ services is encapsulated and mapped into Ethernet frames using Radio over Ethernet (RoE) protocol specified in the IEEE 1914.3 standard [11]. Eventually, time-sensitive networking (TSN) features specified in Reference [9] enable prioritized, low-latency transmission of Ethernet frames carrying latency-sensitive fronthaul traffic.

The Ethernet switches located at remote sites, where traffic from local antennas/access points/other sources is aggregated, and a hub site, where certain baseband processing functions are performed and traffic is forwarded towards the network core, will be principally connected by means of high capacity and low delay links. Optical fiber technologies are a first choice for deployment of such links as they satisfy both requirements and provide some additional capabilities, such as wavelength division multiplexing (WDM). WDM increases significantly the capacity of an optical fiber thanks to multiplexing of signals transmitted on different wavelengths using either passive or active WDM equipment installed at the link ends [12]. Passive WDM reduces about 4-6 times the cost of active WDM since it does not use signal amplification and dispersion compensation components, in addition to the fact that it utilizes less expensive WDM equipment [13]. Therefore, passive WDM solutions are often preferred in limited-distance applications (up to $20 \mathrm{~km}$ ), such as $5 \mathrm{G}$ access networks.

WDM enables aggregation of traffic from remote sites by means of OADM devices, which combine selected wavelengths at particular intermediate sites into an optical transmission path going from an end remote site through intermediate sites to a hub site, as shown in Figure 1. The application of an OADM at an intermediate site increases utilization of the WDM link, and, at the same time, it releases from the need to establish a dedicated transmission path between the hub and the site. It leads to the reduction of the number of fiber connections and installed WDM equipment, which translates into lower network deployment costs [14-16]. Recently, a commercial solution available under the trade name flexiHaul has been proposed for a 5G packet-optical xHaul access network. This solution consists of a TSN Ethernet switch [17] and a passive WDM system xWave 400G [16], which allows for the aggregation of wavelengths on optical paths by means of OADMs. 


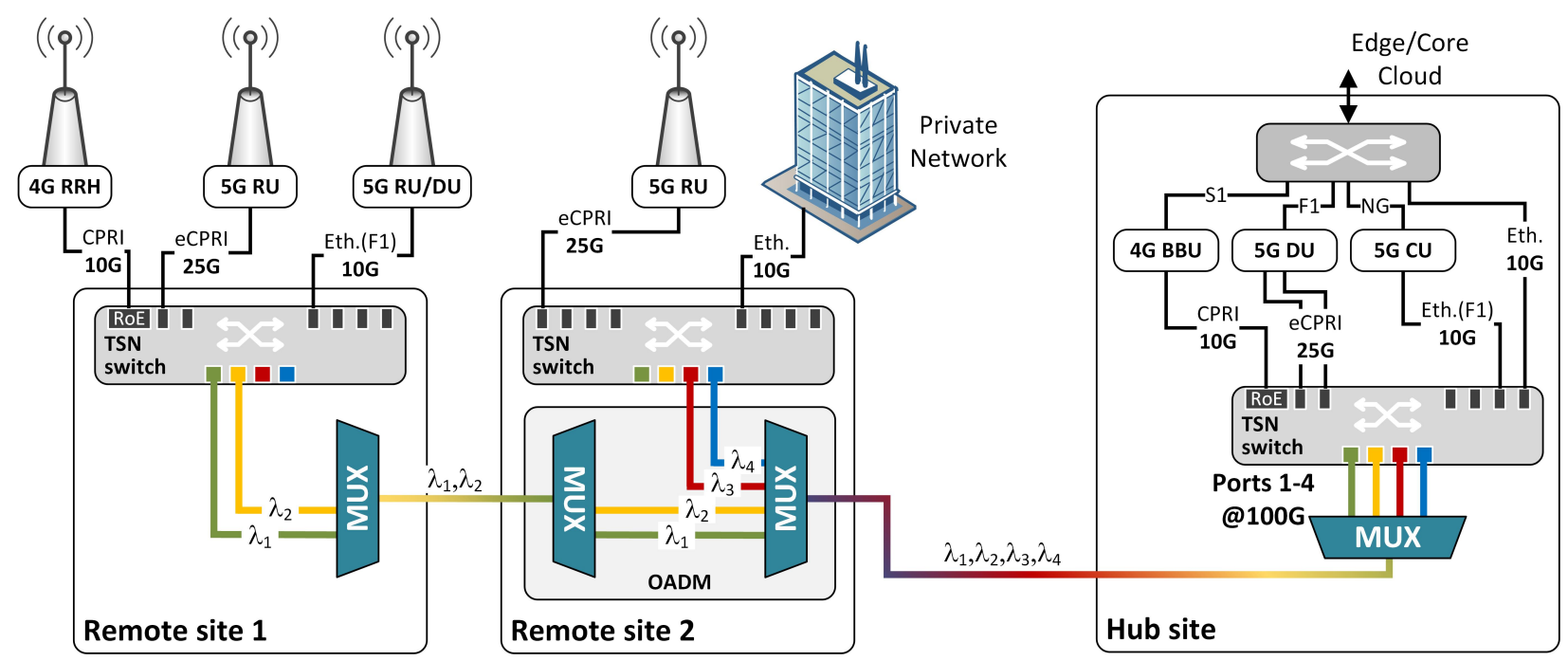

Figure 1. Example of packet-optical xHaul access network carrying 4G, 5G, and private network traffic between a hub and two remote sites (one transmission direction depicted in the optical layer).

In a $5 \mathrm{G}$ access transport network based on a transmission system with the above discussed features, a basic network design problem concerns planning of optical connections between remote sites and a hub site. The problem consists of the selection of routes for optical transmission paths over the network, together with the selection of intermediate nodes at which OADMs are located and traffic is aggregated. These decisions are constrained by the optical power budget of the system, which determines the maximum length of optical paths (i.e., transmission reach/distance). Moreover, the use of OADMs introduces losses of optical power, which results in a shortened transmission reach. As a consequence, there is a certain tradeoff that limits the use of many OADMs on longer paths, although such paths might pass through and allow for gathering traffic from a larger number of remote sites. As aggregation of traffic is desirable for decreasing network cost; hence, proper routing and OADM placement decisions are required when planning optical connections in the network. The length of routing paths and resulting propagation delays may be limited additionally due to low-latency requirements of specific $5 \mathrm{G}$ services.

In this work, we focus on modeling and optimal solving the discussed connection planning problem in a $5 \mathrm{G}$ packet-optical xHaul access network. The main contributions of this work are the following:

1. development and application of a physical-layer transmission model, based on optical power budget calculations and assuming the properties of a passive WDM system, for estimating the reach of transmission paths,

2. formulation of an ILP optimization problem for generation of optimal solutions to the connection planning problem in the $5 \mathrm{G}$ packet-optical $\mathrm{xHaul}$ access network considered, and

3. assessment of the impact of wavelength aggregation (by means of OADMs) on network performance in different scenarios assuming realistic transmission system parameters.

To the best of our knowledge, the optimization problem addressed and the ILP formulation proposed have not been considered in the literature yet. In addition, we are not aware of a similar work in which a physical-layer transmission model was included to optimization of a 5G packet-optical xHaul access network.

The remainder of this article is organized as follows. In Section 2, we discuss related works. In Section 3, we present main assumptions concerning the transmission model and latency constraints. In Section 4, we formulate the optimization problem and model it as an ILP problem. In Section 5, we report the results of numerical experiments. Finally, in Section 6, we conclude this work. 


\section{Related Works}

Optimization of C-RANs connected using optical fiber networks has been frequently addressed jointly with the problem of placement of BBU/DU/CU processing resources. In Reference $[14,15]$, the authors studied a BBU placement problem in a C-RAN connected using a WDM optical network. The optimization problem was formulated as an ILP problem with the objective to minimize the total network cost represented by either the number of active BBU sites or the number of fibers used to transport the traffic in the network. In the study, the underlying optical network was given, and a generic WDM system was considered, in which transmission distance of optical paths (lightpaths) was not constrained by a physical-layer transmission model. The authors of Reference [18] proposed an ILP formulation for the problem of dimensioning of BBU processing and optical transponder resources in a CPRI-based C-RAN connected using an active WDM optical network equipped with sliceable bandwidth-variable transponders. In that work, dedicated point-to-point connections were assumed, without traffic aggregation, and there were not any transmission reach-related constraints. The problem of designing dense WDM (DWDM) rings in metro and access segments of a survivable 5G transport network was studied in Reference [19]. The authors proposed different schemes for survivable $5 \mathrm{G}$ transport and made use of a heuristic approach for planning and dimensioning of ring-based fiber connections. In Reference [20], the BBU location problem with planning of survivable (i.e., primary and backup) lightpath connections in a 5G fronthaul network was addressed. In that work, a generic WDM optical network without any constraints on transmission distance was assumed. The authors of Reference [21] focused on ILP modeling of the problem of DU and CU placement with lightpath provisioning in a ring-based WDM metro/aggregation network. Similar as in other aforementioned works, a generic WDM system was assumed, and transmission reach was not modeled. A literature survey on resource allocation-related problems and solutions in centralized RANs can be found [22].

Different solutions have been considered for passive WDM networks. Coarse wavelength division multiplexing (CWDM) has been widely used in local and metropolitan networks. In CWDM, the channel spacing equals $20 \mathrm{~nm}$, which enables the use of 18 channels in the wavelength range from $1271 \mathrm{~nm}$ to $1611 \mathrm{~nm}$. Due to the wide range of dispersion, CWDM has limited use in systems with an extreme bit rate of $100 \mathrm{~Gb} / \mathrm{s}$ per wavelength. Zero dispersion of the G.652D fiber [23] lies in the range of $1312 \pm 12 \mathrm{~nm}$. For this reason, in the IEEE 802.3cu standard [24], the wavelength range is limited to 4 channels: 1271, 1291, 1311, and $1331 \mathrm{~nm}$. A two times denser spacing, i.e., $10 \mathrm{~nm}$, was proposed in the MWDM system [13] - 12 channels were located in the range from 1267.5 to $1374.5 \mathrm{~nm}$. In addition, 12-channel LAN-WDM system (LWDM) with an $800 \mathrm{GHz}$ inter-channel spacing is used, which, in the $\mathrm{O}$ transmission window, corresponds to a spacing of about $4.5 \mathrm{~nm}$ [13] and the center of channels passband equals, respectively, 1269.23, 1273.55, 1277.89, 1282.26, 1286.66, $1291.10,1295.56,1300.05,1304.58,1309.14,1313.73$, and $1318.35 \mathrm{~nm}$. Recently, an 8-channel system with a $400 \mathrm{GHz}$ channel spacing, i.e., about $2.25 \mathrm{~nm}$ in the $\mathrm{O}$ transmission window (called nWDM-narrow WDM) has been proposed [16], in which the center of channels passband equals, respectively, 1295.56, 1297.80, 1300.05, 1302.31, 1304.58, 1306.85, 1309.14, and $1311.43 \mathrm{~nm}$. This system, under the trade name of flexiHaul xWave 400G, offers a $20 \mathrm{~km}$ transmission distance and a $17 \mathrm{~dB}$ power budget. Four-level amplitude modulation (PAM-4) is used, which enables two bits of information to be encoded in one code symbol. Symbols are transferred at 53.125 Gbaud. Forward error correction RS $(544,514)$ is applied to allow error-free transmission for the input bit error rate less than $2.4 \times 10^{-4}$ [25]. In the mentioned WDM systems, multiplexers (MUX), demultiplexers (DMUX), and add/drop modules (OADM), utilizing optical thin-film filters (TFF), are used to extract a channel of a specific wavelength. They have better transmission properties than Bragg grids (FBG) and array waveguide gratings (AWG), especially with inter-channel spacing greater than $200 \mathrm{GHz}[26]$. 


\section{Main Assumptions}

In this section, we present the details of the optical transmission system and transmissionreach model considered in this study. Moreover, we discuss the assumptions that we take concerning the latencies of transported radio data and their impact on the transmission path length.

\subsection{Transmission Model}

In this article, we assume the properties of WDM transmission system xWave 400G [16] applied in the xHaul 5G optical-packet access network. As discussed in Section 2, the system is characterized by a narrow inter-channel spacing. The benefit of a small inter-channel spacing is the reduction of signal distortion caused by dispersion, while the drawback is the need to use lasers with low chirp, temperature drift in the transmitter, and greater nonlinear distortions. In this case, the limitation of the transmission distance is caused by the power budget. Therefore, we present a detailed model for estimating this budget in the following section.

Table 1 shows the system parameters required to calculate the transmission distance $L$ expressed by Equation (1), determined on the basis of the power and attenuation budget. The number of OADMs introduced into the optical path is denoted by $N$.

$$
L=\min \left(\frac{P_{\mathrm{TXoma}}-P_{\mathrm{RXoma}}-4 N A_{\mathrm{C}}-2 N A_{\mathrm{MUX}}-A_{\mathrm{MM}}}{\alpha}, L_{\max }\right)[\mathrm{km}] .
$$

Table 1. Transmission system parameters.

\begin{tabular}{cc} 
Fiber loss coefficient $(\alpha)[\mathrm{dB} / \mathrm{km}]$ & 0.5 \\
Connector loss $\left(A_{\mathrm{C}}\right)[\mathrm{dB}]$ & 0.25 \\
Maximum number of carried wavelengths $(W)$ & 4 \\
MUX4 loss $\left(A_{\mathrm{MUX}}[\mathrm{dB}]\right.$ & $1.8 \times\{1.0,0.9,0.8\}$ \\
Maintenance margin $\left(A_{\mathrm{MM}}\right)[\mathrm{dB}]$ & 1.0 \\
Minimal transmitter outer optical modulation amplitude & 3.0 \\
$\left(P_{\mathrm{TXoma}}\right)[\mathrm{dBm}]$ & -14.0 \\
Receiver sensitivity $\left(P_{\mathrm{R} X \mathrm{oma}}\right)[\mathrm{dBm}]$ & 20.0 \\
Maximum transmission distance $\left(L_{\max }\right)[\mathrm{km}]$ & \\
\hline
\end{tabular}

Minimal transmitter outer optical modulation amplitude $\left(P_{\text {TXoma }}\right)$ is defined as the minimal difference between two outer (maximal and minimal) optical power levels in PAM4 signal at transmitter output. Receiver sensitivity $\left(P_{\mathrm{RXoma}}\right)$ is defined as the difference between two outer (maximal and minimal) optical power levels in PAM-4 signal at receiver input sufficient to obtain bit error rate less than $2.4 \times 10^{-4}$ before FEC correction. Power budget is the difference between $P_{\mathrm{TX}}$ oma and $P_{\mathrm{RX}}$ oma (see Reference [25], Figure 2-1).

The G.652D fiber loss coefficient value of $0.40 \mathrm{~dB} / \mathrm{km}$ given in Reference [23] is increased here by $0.10 \mathrm{~dB} / \mathrm{km}$ taking into account additional splices losses and environmental margin. To calculate the influence of connectors on attenuation budget, we exploit a statistical approach due to a large number of connectors utilized $(4 N)$, and, as a consequence, mean value represents connector loss. The maximum transmission distance $\left(L_{\max }\right)$ of the flexiHaul xWave 400G system is limited to $20 \mathrm{~km}$ [16].

MUX and DEMUX modules always operate in pairs, so, if they have the same order of filters, the total attenuation we estimated is at approximately $2 / 3$ of combined maximum MUX/DEMUX attenuations (e.g., $3.6 \mathrm{~dB}$ instead of $5.4 \mathrm{~dB}$ ) because the channel added in the MUX farthest from the output is the most attenuated, while, in the DMUX, it is dropped first and the least attenuated. This applies to both the MUX/DEMUX pair which is part of OADM (see Figure 1) and to the first/last MUX/DEMUX pair in each optical path. Attenuation of cut-through OADM optical path is increased by patchcord attenuation equal to $2 \times A_{C}$.

Figure 2 shows the system configuration with indicated parameters necessary to estimate the transmission distance from Equation (1). A minimum of one wavelength is 
dedicated to a single remote site, so the maximum remote sites number equals $W$. In the information available to us about the parameters of the flexiHaul xWave 400G system, there is no exact data for the attenuation of MUX and OADM modules. Therefore, the budget calculations are based on the parameters of similar, off-the-shelf components, made in the same TFF technology, available from various manufacturers $[27,28]$; hence, we use several variants for values of $A_{\mathrm{MUX}}$ adopted in the analysis.

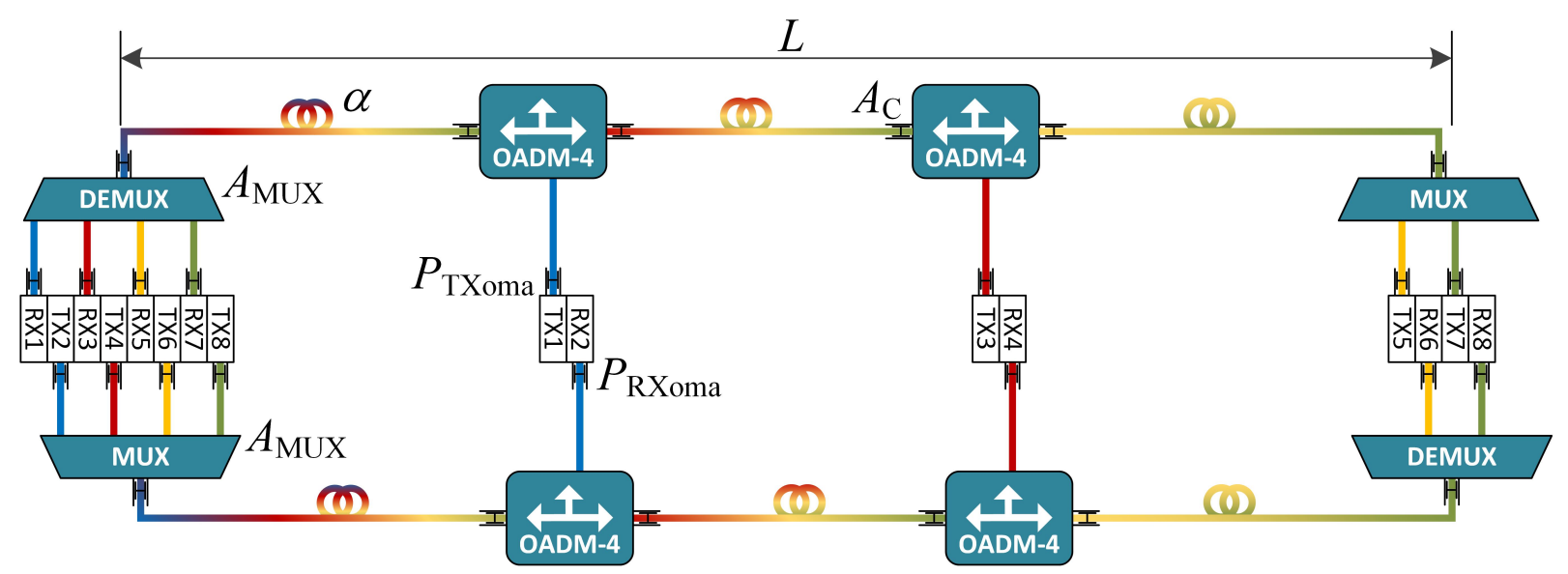

Figure 2. System configuration with denoted parameters necessary to estimate the transmission distance $L$.

Eventually, in Table 2, we show the transmission distance $(L)$ values obtained using Equation (1) and system parameters presented in Table 1, for different values of MUX loss and the number of OADMs present on the optical transmission path. The obtained values of $L$ are used in the optimization model presented in the next section to determine the maximum number of OADMs that can be installed on a transmission path of a given length.

Table 2. Transmission distance $L$ as a function of MUX loss and number of installed OADMs.

\begin{tabular}{ccccc}
\hline \multirow{2}{*}{ MUX Loss } & \multicolumn{4}{c}{ Number of OADMs $(\mathbf{N})$} \\
\cline { 2 - 5 } & $\mathbf{0}$ & $\mathbf{1}$ & $\mathbf{2}$ & $\mathbf{3}$ \\
\hline $1.80 \mathrm{~dB}$ & $20.00 \mathrm{~km}$ & $13.60 \mathrm{~km}$ & $4.40 \mathrm{~km}$ & - \\
$1.62 \mathrm{~dB}$ & $20.00 \mathrm{~km}$ & $15.04 \mathrm{~km}$ & $6.56 \mathrm{~km}$ & - \\
$1.44 \mathrm{~dB}$ & $20.00 \mathrm{~km}$ & $16.48 \mathrm{~km}$ & $8.72 \mathrm{~km}$ & $0.96 \mathrm{~km}$ \\
\hline
\end{tabular}

\subsection{Latency Constraints}

The technical specification of the Ethernet switch utilized in the flexiHaul system [17] mentions the implementation of the TSN mechanisms defined by standards IEEE 802.1CM and IEEE 802.1Qbu. We assume that these TSN mechanisms are applied and they assure the prioritized transmission of Ethernet frames carrying latency-sensitive data, in particular, related to 5G fronthaul traffic. Still, under heavy fronthaul traffic loads, the latencies of higher priority frames may be increased due to the need for their buffering since they compete each other for transmission resources at the switch output link. To account for this effect, we consider that the length of transmission paths between remote sites and the hub is constrained additionally by the latency requirements of latency-sensitive data flows.

Namely, in the analysis performed in this work, we consider that the one-way latency budget of latency-sensitive flows is equal to $100 \mu$ s (according to Reference [29]). Within this budget, we assume that $50 \mu$ s is reserved, among others, for the store-and-forward operations in the switch (a few $\mu$ s, as mentioned in Annex B in Reference [9]), the transmission time of frames (not exceeding a few $\mu$ s in a 100 Gbps optical link [30]), and queuing latency (up to some tens of $\mu \mathrm{s}$ ), whereas the remaining $50 \mu \mathrm{s}$ is left for signal propagation in the optical fiber. Note that, in the transmission system studied in this work, the buffering of 
frames in the xHaul network occurs once for each transmission direction, namely during aggregation of radio data flows into wavelength connections at the remote TSN switch and at the switch located in the hub, respectively, for uplink and downlink direction. Assuming a periodic traffic model [31], in which the radio data frames are transmitted periodically and within a certain transmission window of the size of up to some tens of $\mu \mathrm{s}$, the queuing latency does not exceed the transmission window duration in the worst case, in which all HP frames arrive at the same moment, and the link capacity is fully utilized.

The above assumed $50 \mu$ s propagation delay limit corresponds to the maximum optical path length equal to $10 \mathrm{~km}$. The numerical results presented in Section 5 are obtained considering this limit.

\section{Optimization Problem}

The packet-optical xHaul access network planning problem addressed in this paper consists of finding a set of transmission paths between a given set of remote sites and a hub site. A transmission path is realized either as a direct fiber connection between the end sites or a fiber connection going through OADM devices installed at some remote sites (as shown in Figures 1 and 2). Each transmission path carries a number of wavelengths. An OADM allows for joining into an existing transmission path the wavelengths originated at the remote node at which this device is installed (see $\lambda_{3}$ and $\lambda_{4}$ in remote site 2 in Figure 1). The connections are constrained by: (a) the allowable number of OADMs traversed by a transmission path, which is limited by the optical power budget of the path (as discussed in Section 3.1), and (b) the maximum number of carried wavelengths that must not exceed the capacity of given WDM system. The use of OADMs allows for decreasing the demand for fiber connections in the network. In this work, we aim at optimizing the configurations of transmission paths, namely their routes and placement of OADMs, to minimize the number of fiber connections required in the network.

We begin by introducing the notions and notation used in problem modeling. Afterwards, we formulate the connection planning problem as an ILP optimization problem.

\subsection{Notation}

The xHaul transport network is modeled by a graph $\mathcal{G}=(\mathcal{V}, \mathcal{E})$, where $\mathcal{V}$ denotes the set of network nodes, representing remote and hub sites, and $\mathcal{E}$ is the set of links representing fiber connectivity between the nodes. We assume that the capacity of each link in terms of available fibers is not a bottleneck and is sufficient to carry the traffic between remote and hub nodes. Let $W$ denote the capacity of the WDM system, i.e., the maximum number of wavelengths carried in a fiber.

Let $\mathcal{D}$ be the set of demands. Each demand $d \in \mathcal{D}$ represents a number of wavelength connections to be established in the xHaul network between a remote node and a hub node. We assume that each remote node generates a demand. Let $W(d)$ be the number of requested wavelengths of demand $d$.

Let $\mathcal{P}(d)$ denote the set of candidate transmission paths of demand $d \in \mathcal{D}$. Transmission path $p \in \mathcal{P}(d)$ is a fiber route through the network between the remote node corresponding to demand $d$ and a hub node; in particular, $p$ is a subset of network links $(p \subseteq \mathcal{E})$. Let $D(p)$ denote the physical length of transmission path $p$. We assume that path $p$ may either: (a) realize a direct fiber connection between the end nodes of a demand or (b) be a fiber connection traversing OADM devices installed at some intermediate remote nodes. Let $N(p)$ denote the number of OADMs allowable on path $p . N(p)$ depends on path length $D(p)$ and is determined by the transmission distance values presented in Table 2, namely $N(p)=\max \{N: D(p) \leq L(N)\}$, where $L(N)$ is the value of $L$ for given $N$ in Table 2. Let $\mathcal{Q}(d, \bar{d})$ be the set of candidate transmission paths of demand $\bar{d}$ (i.e., $\mathcal{Q}(d, \bar{d}) \subseteq \mathcal{P}(\bar{d})$ ) which may go through an OADM installed at the remote node of demand $d$. The notation is summarized in Table 3 . 
Table 3. Notation.

\begin{tabular}{ll}
\hline Sets and Parameters \\
\hline $\mathcal{V}$ & set of remote and hub nodes \\
$\mathcal{E}$ & set of links connecting the nodes \\
$\mathcal{D}$ & set of demands \\
$\mathcal{P}(d)$ & set of candidate transmission paths of demand $d \in \mathcal{D}$ \\
$\mathcal{Q}(d, \bar{d})$ & set of candidate transmission paths of demand $\bar{d}$ which can serve demand $d$ \\
$D(p)$ & length of transmission path $p$
\end{tabular}

\subsection{ILP Formulation}

To model the optimization problem, we introduce a set of decision variables. Namely, binary variable $x_{d p}, d \in \mathcal{D}, p \in \mathcal{P}(d)$ indicates whether transmission path $p$ is established for demand $d$. Binary variable $y_{d}, d \in \mathcal{D}$ indicates whether demand $d$ is served by a transmission path established for some other demand, which is achieved by means of an OADM device introduced into the path. Eventually, binary variable $y_{d \bar{d}}, d \in \mathcal{D}, \bar{d} \in \mathcal{D}$ indicates whether demand $d$ is served by a transmission path established for demand $\bar{d}$. The meaning of variables is also summarized in Table 3.

The ILP formulation of the connection planning problem considered is the following:

$$
\begin{aligned}
\operatorname{minimize} z=A \cdot \sum_{d \in \mathcal{D}} x_{d p}+\sum_{d \in \mathcal{D}} \sum_{p \in \mathcal{P}(d)} D(p) \cdot x_{d p}, & \\
\sum_{p \in \mathcal{P}(d)} x_{d p}+y_{d}=1, \quad & \forall d \in \mathcal{D}, \\
\sum_{\bar{d} \in \mathcal{D}, \bar{d} \neq d} y_{d \bar{d}}=y_{d}, & \forall d \in \mathcal{D}, \\
\sum_{p \in \mathcal{Q}(d, \bar{d})} x_{\bar{d} p} \geq y_{d \bar{d}}, & \forall d, \bar{d} \in \mathcal{D}, \bar{d} \neq d, \\
W(d) \cdot\left(1-y_{d}\right)+\sum_{\bar{d} \in \mathcal{D}, \bar{d} \neq d} W(\bar{d}) \cdot y_{\bar{d} d} \leq W, & \forall d \in \mathcal{D}, \\
\sum_{\bar{d} \in \mathcal{D}, \bar{d} \neq d} y_{\bar{d} d} \leq \sum_{p \in \mathcal{P}(d)} N(p) \cdot x_{d p}, & \forall d \in \mathcal{D} .
\end{aligned}
$$

Optimization objective (2) aims at minimizing the number of transmission paths, which is a primary goal imposed by coefficient $A$ (we consider $A=1000$ ), and the overall length of used fibers (a secondary optimization goal). Constraints (3) assure that either a transmission path, selected from the set of candidate paths, is established for a demand $\left(y_{d}=0\right)$ or the demand is served by the transmission path established for some other demand by means of an OADM device $\left(y_{d}=1\right)$. Constraints (4) determine which other demand serves on its transmission path the demand that has not established its own path (i.e., when $y_{d}=1$ ). Constraints (5) assure that, for demands supporting some other demands, their own transmission paths should exist. Constraints (6) assure that the sum of requested wavelengths of a demand for which a transmission path is established (when $y_{d}=0$ ) and the requests wavelengths of other demands served by this transmission path (when $y_{\bar{d} d}=1$ ) must not exceed the fiber capacity (expressed by W). Finally, Constraints (7) 
assure that the number of other demands served by an established transmission path does not exceed the allowable number of OADMs traversed by the path (expressed by $N(p)$ ).

\section{Numerical Results}

In this section, we apply the ILP optimization model presented in Section 4 in network analysis. The evaluation is performed in two network topologies of different size: a 17-node city network (WRO17) and a 38-node mesh network (MESH38), shown in Figure 3. Topology WRO17 was developed based on a subset of real antenna locations (marked by triangles in Figure 3) in the center of city Wroclaw in Poland, where remote sites (marked by circles) are placed in proximity of antennas and connected using links driven along streets. The lengths of links in WRO17 reflect real physical lengths of depicted connections. In reference topology MESH38, which was used in C-RAN studies in Reference [20], we consider that link lengths are uniformly distributed between 1 and 3 kilometers. In both topologies, the hub site is denoted by a hexagonal. The routes of candidate transmission paths between remote nodes and the hub site have been generated using a $k$-shortest path algorithm. The paths of the length exceeding the maximum allowable path length (i.e., $10 \mathrm{~km}$ as discussed in Section 3.2) were excluded from the generated sets of candidate paths. In Table 4, we present some link and path-related statistics corresponding to the topologies.
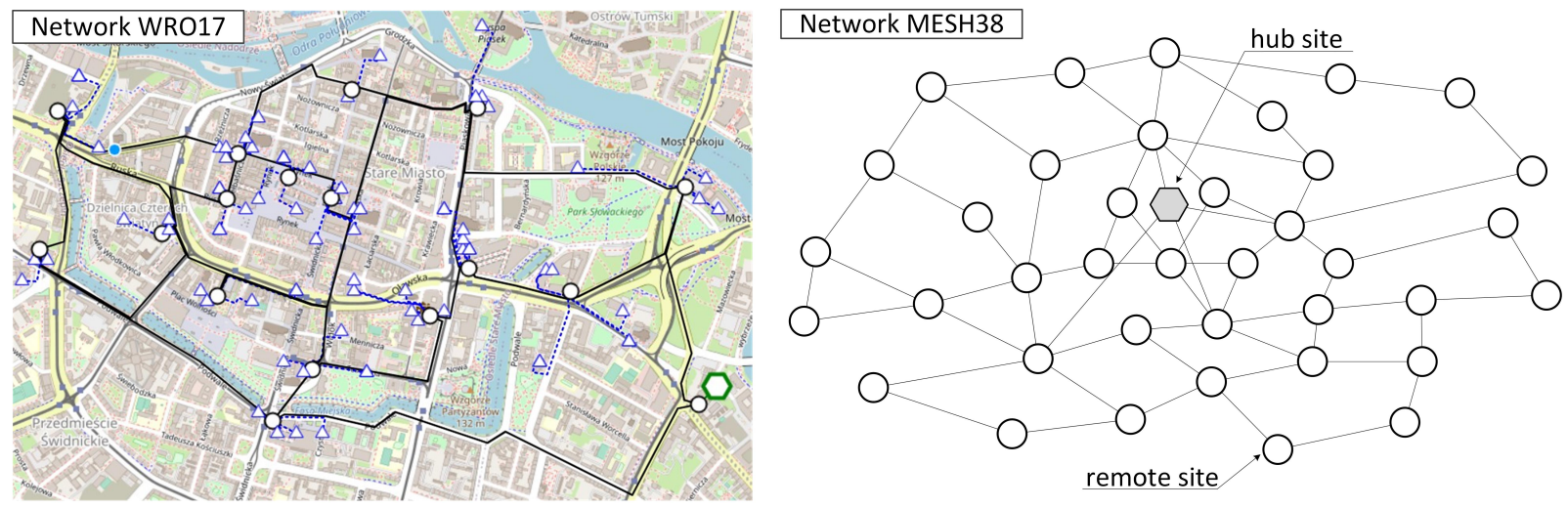

Figure 3. WRO17 and MESH38 network topologies showing fiber connectivity between remote sites (circles) and hub site (hexagon); the triangles in WRO-17 represent real antenna locations.

Table 4. Characteristics of analyzed networks; SP denotes the shortest path.

\begin{tabular}{ccccccccc}
\hline \multirow{2}{*}{ Network } & \multirow{2}{*}{ Remote Sites } & \multirow{2}{*}{ Links } & \multicolumn{2}{c}{ Link length $[\mathbf{k m}]$} & \multicolumn{2}{c}{ SP length $[\mathrm{km}]$} & \multicolumn{2}{c}{ SP hops } \\
\cline { 4 - 9 } & & & Mean & Max & Mean & Max & Mean & Max \\
\hline WRO17 & 17 & 25 & 0.54 & 1.55 & 2.03 & 3.01 & 3.7 & 6 \\
MESH38 & 38 & 64 & 2.12 & 2.92 & 4.38 & 8.42 & 2.5 & 4 \\
\hline
\end{tabular}

As mentioned in Section 4, each remote site requests a certain number of wavelengths to be carried towards the hub site. The number of wavelengths requested by a remote site is generated randomly with a uniform distribution between $N^{\min }$ and $N^{\max }$. Traffic load, denoted as $\rho$, is defined as the average number of requested wavelengths per remote site, namely $\rho=\left(N^{\min }+N^{\max }\right) / 2$. We evaluate different traffic scenarios, where $N^{\min } \geq 1$ and $N^{\max } \leq 4$. In particular, we have $\rho=1.0$ for $N^{\min }=N^{\max }=1 ; \rho=1.5$ for $N^{\min }=1, N^{\max }=2 ; \rho=2.0$ for $N^{\min }=1, N^{\max }=3$, etc. In each traffic scenario, the results are obtained and averaged over 10 randomly generated demand sets.

We assume the transmission model and system parameters discussed in Section 3.1. In particular, we evaluate the impact of MUX loss $A_{\mathrm{MUX}}$ on network performance, where $A_{\mathrm{MUX}} \in\{1.8,1.62,1.44\} \mathrm{dB}$. As a reference scenario, denoted as no-OADMs, we consider the network in which OADMs are not used and dedicated transmission paths are estab- 
lished between every remote node and the hub. In particular, we have 17 and 38 such transmission paths in WRO17 and MESH38, respectively.

The numerical experiments are performed on a $3.7 \mathrm{GHz}$ 32-core Ryzen Threadripperclass machine with 64 GB RAM. To solve the ILP model, we use CPLEX v.12.9 solver [32]. All the results are optimal and computation times of CPLEX do not exceed $80 \mathrm{~s}$ in the most demanding scenario.

In Figure 4, we illustrate optimal transmission paths found in network WRO17 for two selected traffic scenarios with loads $\rho=1.0$ and $\rho=2.0$, assuming MUX attenuation $A_{\mathrm{MUX}}=1.8 \mathrm{~dB}$ and $k=3$ candidate routing paths (no impact on results were observed for $k>3$ in WRO17). The paths, as well as the remote nodes making use of the paths, are marked with different colors. For instance, one of the paths in the left-side figure begins in node 8 and goes through intermediate nodes 3 and 0 , where some wavelengths are introduced into the path by means of OADMs, and finally terminates in the hub site attached to node 2 . We can see that six optical paths are sufficient to carry traffic load $\rho=1.0$, whereas two more paths (eight paths in total) are required to support scenario $\rho=2$.0. This difference is a result of a higher number of wavelengths requested at certain sites in the latter scenario which cannot be served due to a limited capacity of the WDM transmission system (4 wavelengths). Note that there are also some differences in the placement of OADMs (i.e., the assignment of remote nodes to the paths) in both scenarios. For instance, node 12 is either an intermediate node of the orange path in scenario $\rho=1.0$ or an end node of the light blue path in scenario $\rho=2.0$.
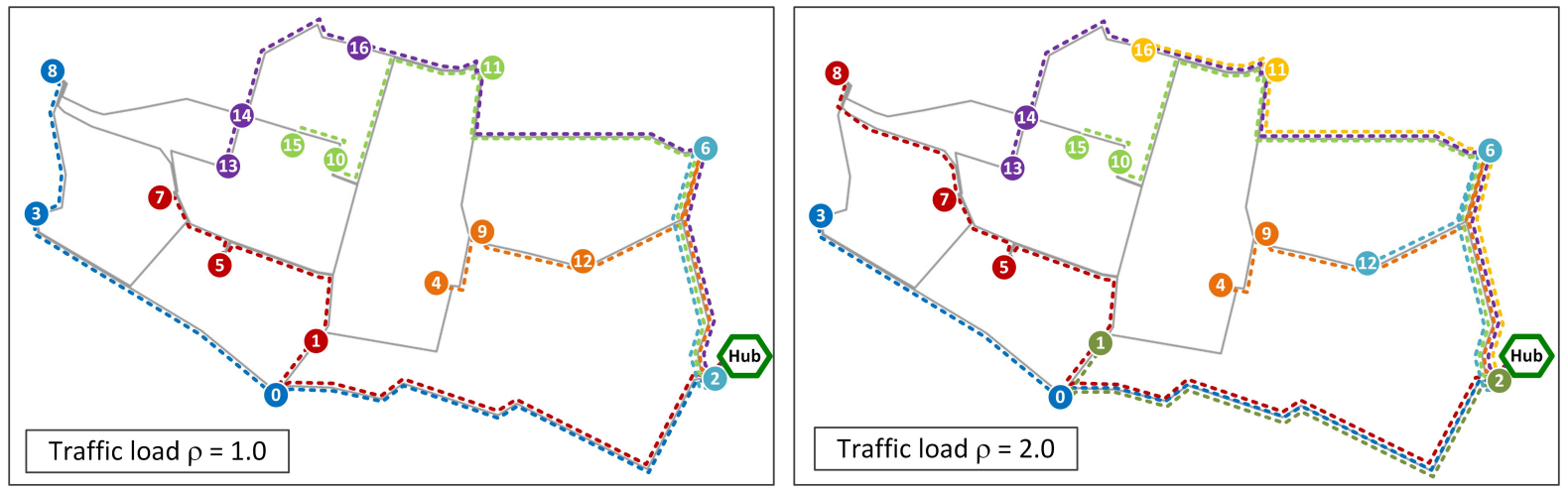

Figure 4. Optimal solutions obtained in WRO17 for traffic load $\rho=1.0$ (left) and $\rho=2.0$ (right).

In Figure 5, we present averaged results of the number of transmission paths (left chart) and overall length of transmission paths (right chart) in a function of traffic load $(\rho)$ in WRO17, assuming MUX loss $A_{\mathrm{MUX}}=1.8 \mathrm{~dB}$ and $k=3$ candidate paths. Additionally, in both figures, we show a relative difference (gain) in the obtained results when compared to the noOADM reference scenario.

We can see that 6 transmission paths are sufficient to serve all traffic in the network when each remote node generates a 1-wavelength demand (i.e., $\rho=1.0$ ). This is achieved by aggregation of wavelengths (using OADMs) from different remote sites onto WDM optical transmission paths. In this case, the reduction of the number of required transmission paths (i.e., relative gain) versus the scenario without OADMs reaches about $65 \%$. Increasing the traffic load, the number of transmission paths increases (and the relative gain decreases) up to the moment when all remote nodes need dedicated transmission paths. It happens when 3.5 wavelengths, on average, are requested by each remote node $(\rho=3.5)$, and there is no use of OADMs due to saturation of the WDM system, which capacity is 4 wavelengths. The overall length of optical transmission paths (shown in the right chart) follows a similar trend. In particular, the length of dedicated fiber connections required in the noOADM scenario is about $34.5 \mathrm{~km}$, which can be reduced by up to $60 \%$ (to about $14 \mathrm{~km}$ ) in a network with a low load $(\rho=1.0)$. We report that similar results were obtained for other considered values of $A_{\mathrm{MUX}}$. 

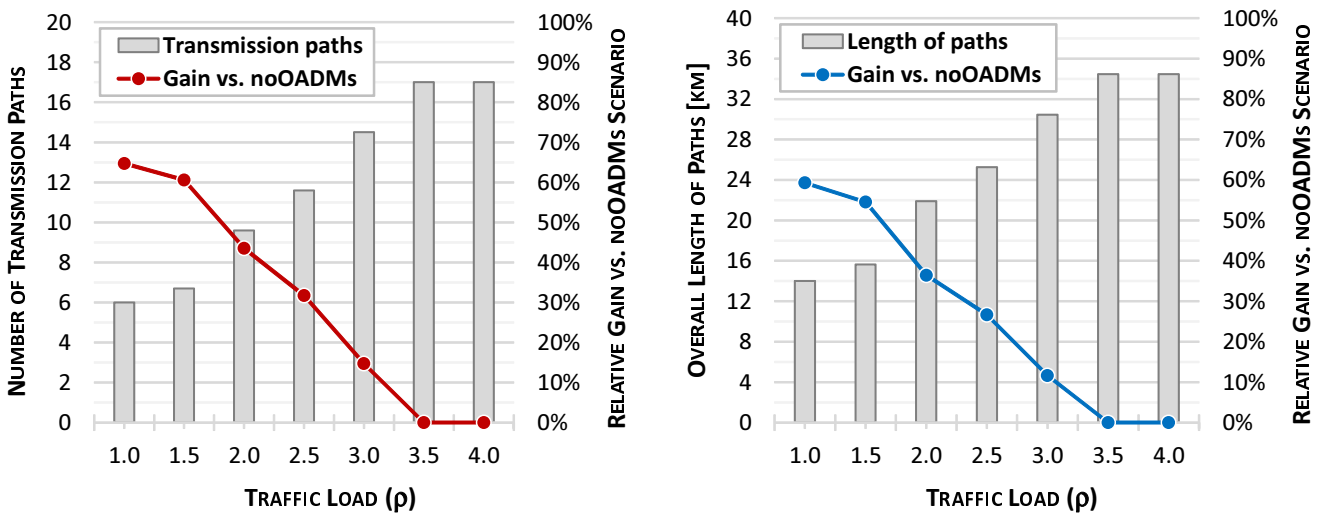

Figure 5. The number of transmission paths (left) and overall length of paths (right) in a function of traffic load $(\rho)$ in WRO17; relative gain versus the network without OADMs is shown as lines.

In Figure 6, we analyze the impact of the number of candidate routing paths $k$ on obtained results in network MESH38. We focus on the number of optical transmission paths (left chart) in a function of $k$ and traffic load, $\rho \in\{1.0,1.5,2.0\}$, for different values of MUX loss ( $\left.A_{\mathrm{MUX}}\right)$. Moreover, in the right chart, we show a relative difference in results when compared to a single (shortest) path scenario $(k=1)$. In each scenario, we can see that provisioning of a higher number of candidate routes $(k>1)$ allows for reducing the number of optical transmission paths required in the network when compared to the shortest-path case. The gain in performance increases with $k$ and ranges between $16 \%$ and $32 \%$, depending on traffic and MUX scenario, for $k \geq 5$. We can also see that the results stabilize for $k \geq 5$ and that the improvement for $k=7$ is either none or irrelevant (as for $\rho=2.0$ ). Finally, higher differences in obtained results are observed for lower MUX loss values at lower traffic loads. It can be explained by higher transmission distances and numbers of allowable OADMs (as shown in Table 2) in low MUX loss scenarios, which translates into a higher chance to inject single wavelengths into transmission paths at intermediate nodes, especially if several alternative candidate paths are available.
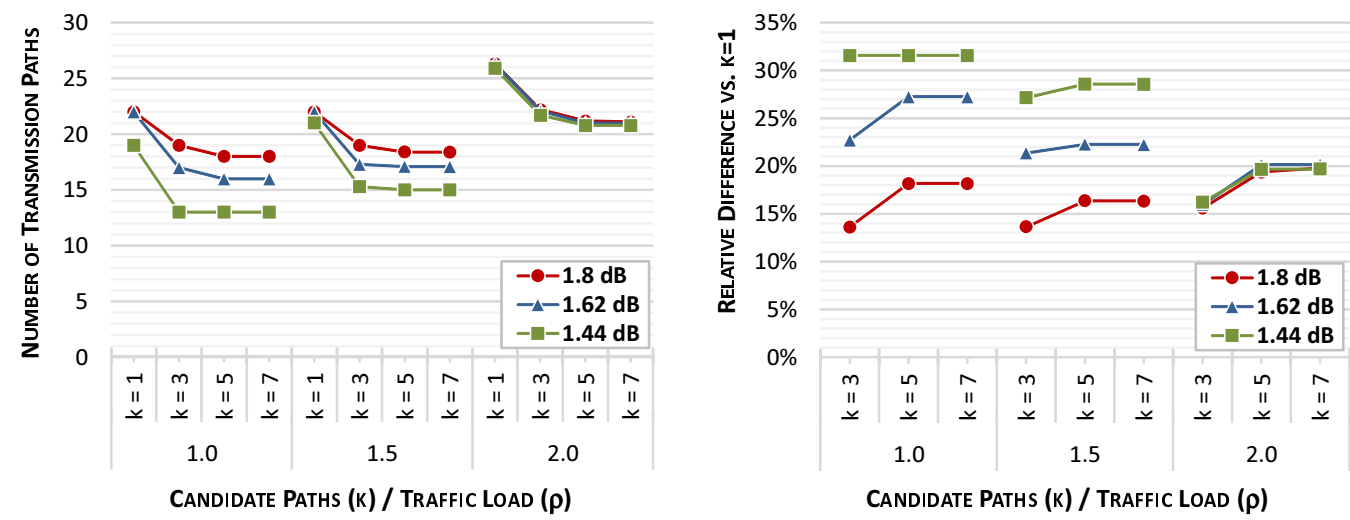

Figure 6. The number of transmission paths (left) and relative difference versus a single-path scenario (right) in a function of traffic load $(\rho)$ and the number of candidate routing paths $(k)$ for different MUX loss values in MESH38.

Eventually, in Figure 7, we show the results of the number of transmission paths (left chart) and the relative performance gain versus reference scenario noOADM (right chart) in a function of traffic load $(\rho)$ in MESH38 for different MUX loss values ( $A_{\text {MUX }}$ ) and assuming $k=7$ candidate paths. We can see that, under low traffic loads $(\rho \leq 1.5)$, the use of MUXs with lower attenuation results in a lower number of required transmission paths. In this case, higher transmission distances are allowable and more OADMs can be used on the paths (as for $A_{\mathrm{MUX}}=1.44 \mathrm{~dB}$ ), which, under a low demand for wavelengths (at 
most 2 wavelengths per remote node), allows for higher aggregation of demands, as also discussed in the above remarks concerning Figure 6. At higher traffic loads $(\rho \geq 2.0)$, the gains from using low-loss MUXes are either none or negligible in the 4-channel WDM system considered, due to its saturation with the carried wavelengths. The gains from using OADMs are between $52 \%$ and $65 \%$ for $\rho=1.0$, depending on the MUX scenario, and are decreasing up to $0 \%$, which is reached at a high load for $\rho=3.5$.
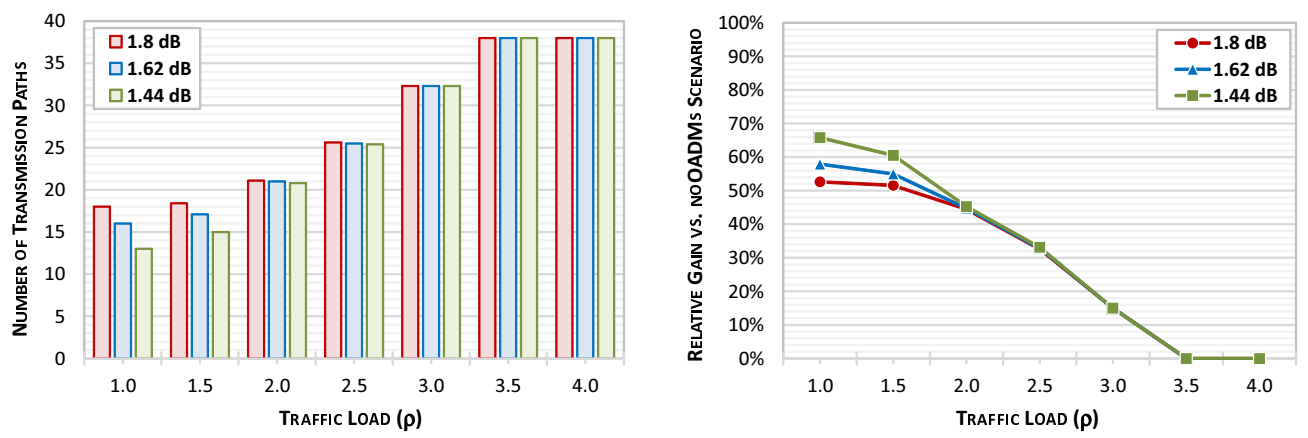

Figure 7. Number of transmission paths (left) and relative gain versus the network without OADMs (right) in a function of traffic load $(\rho)$ for different MUX loss values in MESH38.

\section{Concluding Remarks}

We have studied the problem of planning optimal transmission paths, realized using optical fiber connections and optical add-drop multiplexers, in a 5G packet-optical xHaul access network. The planning problem has concerned the selection of routing paths in the network, between a set of remote nodes and a hub site, and placement of OADMs onto the paths for traffic aggregation with the goal to minimize the number of transmission paths in the network. The mentioned optimization problem was formulated as an integer linear programming problem. To estimate maximum transmission distances and determine the maximum number of OADMs allowable on particular paths, we have developed a transmission model based on optical power budget calculation. In network analysis, we have considered the functionality and parameters of a commercial packet-optical xHaul system and optical components available on the market. The evaluation was performed in two different network topologies, including an urban typology developed based on real antenna locations and realistic transmission distances.

We have shown that the application of OADMs allows to decrease the number of required transmission paths significantly when compared to the network in which aggregation of traffic from remote sites by means of OADMs is not realized. The reduction in the number of transmission paths in both evaluated networks can reach up to $65 \%$ under a low load, which translates into a lower demand for fiber resources and, consequently, cost savings. Even at higher traffic loads, with an average demand corresponding to $75 \%$ of the WDM system capacity, i.e., for 3 wavelengths requested on average per remote site, the savings of about $15 \%$ can be achieved. Provisioning of multiple candidate routing paths, instead of using the shortest path only, is beneficial as it allows for a more effective traffic aggregation and, hence, reduction of the number of transmission paths, between 16\% and $32 \%$, depending on traffic and MUX loss scenario. Finally, the application of MUXs with lower attenuation can be advantageous for low load scenarios; however, under moderate and higher traffic, they have not offered any performance gains in the analyzed networks.

In future works, we will address packet-optical xHaul network scenarios in which diverse types of OADMs are applied instead of a fixed OADM assumed in this work. In addition, we will extend the optimization models to account for WDM systems in which the power loss levels are not the same for particular wavelengths. Eventually, we plan to study multi-layer $\mathrm{xHaul}$ scenarios in which routing decisions concern both the packet and optical layer. 


\begin{abstract}
Author Contributions: Conceptualization, M.K.; Funding acquisition, M.K.; Investigation, M.K. and M.J.; Methodology, M.K. and M.J.; Project administration, M.K.; Software, M.K.; Supervision, M.K.; Visualization, M.K.; Writing—original draft, M.K. and M.J.; Writing—review \& editing, M.K. and M.J. All authors have read and agreed to the published version of the manuscript.

Funding: This research was funded by National Science Center, Poland under grant number 2018/31/B/ST7/03456.
\end{abstract}

Conflicts of Interest: The author declares no conflict of interest.

\title{
References
}

1. Agiwal, M.; Roy, A.; Saxena, N. Next Generation 5G Wireless Networks: A Comprehensive Survey. IEEE Commun. Surv. Tutor. 2016, 18, 1617-1655. [CrossRef]

2. Peng, M.; Sun, Y.; Li, X.; Mao, Z.; Wang, C. Recent Advances in Cloud Radio Access Networks: System Architectures, Key Techniques, and Open Issues. IEEE Commun. Surv. Tutor. 2016, 18, 2282-2308. [CrossRef]

3. The 3rd Generation Partnership Project (3GPP). Available online: http://www.3gpp.org/ (accessed on 29 November 2021).

4. IEEE. 1914.1-2019_IEEE Standard for Packet-Based Fronthaul Transport Networks; IEEE: Piscataway, NJ, USA, 2019.

5. Wypiór, D.; Klinkowski, M.; Michalski, I. Open RAN-Radio Access Network Evolution, Benefits and Market Trends. Appl. Sci. 2022, 12, 408. [CrossRef]

6. Aguerri, I.E.; Zaidi, A. Lossy Compression for Compute-and-Forward in Limited Backhaul Uplink Multicell Processing. IEEE Trans. Commun. 2016, 64, 5227-5238. [CrossRef]

7. Park, S.H.; Simeone, O.; Sahin, O.; Shamai, S. Robust and Efficient Distributed Compression for Cloud Radio Access Networks. IEEE Trans. Vehic. Technol. 2013, 62, 692-703. [CrossRef]

8. Aguerri, I.E.; Zaidi, A.; Caire, G.; Shitz, S.S. On the Capacity of Cloud Radio Access Networks with Oblivious Processing. IEEE IEEE Trans. Inf. Theory 2019, 65, 4575-4596. [CrossRef]

9. IEEE. 802.1CM-2018-IEEE Standard for Local and Metropolitan Area Networks-Time-Sensitive Networking for Fronthaul; IEEE: Piscataway, NJ, USA, 2018.

10. Common Public Radio Interface: eCPRI V1.2 Interface Specification. 2018. Available online: http://www.cpri.info/downloads/ eCPRI_v_1_2_2018_06_25.pdf (accessed on 29 November 2021).

11. IEEE. 1914.3-2018_IEEE Standard for Radio over Ethernet Encapsulations and Mappings; IEEE: Piscataway, NJ, USA, 2018.

12. Alimi, I.A.; Teixeira, A.; Monteiro, P. Towards an Efficient C-RAN Optical Fronthaul for the Future Networks: A Tutorial on Technologies, Requirements, Challenges, and Solutions. IEEE Commun. Surv. Tutor. 2018, 20, 708-769. [CrossRef]

13. O-RAN Alliance. WDM-Based Fronthaul Transport Tech. Spec. v1.0. 2021. Available online: https://www.o-ran.org/ (accessed on 29 November 2021).

14. Carapellese, N.; Tornatore, M.; Pattavina, A.; Gosselin, S. BBU Placement over a WDM Aggregation Network Considering OTN and Overlay Fronthaul Transport. In Proceedings of the 2015 European Conference on Optical Communication (ECOC), Valencia, Spain, 27 September-1 October 2015.

15. Musumeci, F.; Bellanzon, C.; Tornatore, N.C.M.; Pattavina, A.; Gosselin, S. Optimal BBU Placement for 5G C-RAN Deployment over WDM Aggregation Networks. IEEE J. Lightw. Technol. 2016, 34, 1963-1970. [CrossRef]

16. HFR Networks. flexiHaul xWave 400G Data Sheet. Available online: https://hfrnetworks.com/wp-content/uploads/2021/04/ HFR-Networks_flexiHaul-xWave-400G_DataSheet_04-13-2021-1.pdf (accessed on 29 November 2021).

17. HFR Networks. flexiHaul Packet M-Series TSN Switch Data Sheet. Available online: https://hfrnetworks.com/wp-content/ uploads/2020/10/M6424-Packet-TSN-RoE-Switch-DS_HFR-Networks_102420.pdf (accessed on 29 November 2021).

18. Velasco, L.; Castro, A.; Asensio, A.; Ruiz, M.; Liu, G.; Qin, C.; Proietti, R.; Yoo, S.J.B. Meeting the Requirements to Deploy Cloud RAN Over Optical Networks. OSA/IEEE J. Opt. Commun. Netw. 2017, 9, B22-B32. [CrossRef]

19. Wong, E.; Grigoreva, E.; Wosinska, L.; Machuca, C.M. Enhancing the Survivability and Power Savings of 5G Transport Networks based on DWDM Rings. OSA/IEEE J. Opt. Commun. Netw. 2017, 9, D74-D85. [CrossRef]

20. Khorsandi, B.M.; Raffaelli, C. BBU location algorithms for survivable 5G C-RAN over WDM. Comput. Netw. 2018, 144, 53-63. [CrossRef]

21. Yu, H.; Musumeci, F.; Zhang, J.; Xiao, Y.; Tornatore, M.; Ji, Y. DU/CU Placement for C-RAN over Optical Metro-Aggregation Networks. In Proceedings of the International IFIP Conference on Optical Network Design and Modeling, Athens, Greece, 13-16 May 2019.

22. Ejaz, W.; Sharma, S.K.; Saadat, S.; Naeem, M.; Anpalagan, A.; Chughtai, N.A. A Comprehensive survey on Resource Allocation for CRAN in 5G and Beyond Networks. J. Net. Comput. Appl. 2020, 160, 102638. [CrossRef]

23. ITU-T. Recommendation G.652: Characteristics of a Single-Mode Optical Fibre and Cable. 2016. Available online: https: / / www.itu.int/rec/T-REC-G.652/en (accessed on 29 November 2021).

24. IEEE. Standard 802.3cu-2021, Amendment 11: Physical Layers and Management Parameters for 100 Gb/s and 400 Gb/s Operation over Single-Mode Fiber at $100 \mathrm{~Gb} / \mathrm{s}$ per Wavelength; IEEE: Piscataway, NJ, USA, 2021.

25. 100G Lambda MSA. 100G-LR1-20, 100G-ER1-30 and 100G-ER1-40 Tech. Spec. Rev 1.1. Available online: http:/ /100glambda.com/ (accessed on 21 January 2022). 
26. Grobe, K. Handbook of Fiber Optic Data Communication, 4th ed.; Academic Press: Cambridge, MA, USA, 2013.

27. FS. 4ch Single Fiber 1295.56-1309.14nm LAN-WDM MUX DEMUX Technical Data. Available online: https://www.fs.com/ (accessed on 21 January 2022).

28. EDGE Technologies Ltd.. 5G Fronthaul WDM. Available online: https://edgeoptic.com/product/5g-fronthaul-wdm/ (accessed on 21 January 2022).

29. Common Public Radio Interface: eCPRI V1.2 Requirements for the eCPRI Transport Network. 2018. Available online: http:// www.cpri.info/downloads/Requirements_for_the_eCPRI_Transport_Network_V1_2_2018_06_25.pdf (accessed on 29 November 2021).

30. Klinkowski, M. Optimization of Latency-Aware Flow Allocation in NGFI Networks. Comp. Commun. 2020, 161, $344-359$. [CrossRef]

31. Perez, G.O.; Larrabeiti, D.; Hernandez, J.A. 5G New Radio Fronthaul Network Design for eCPRI-IEEE 802.1CM and Extreme Latency Percentiles. IEEE Access 2019, 7, 82218-82229. [CrossRef]

32. IBM. CPLEX Optimizer. Available online: https://www.ibm.com/analytics/cplex-optimizer (accessed on 29 November 2021). 